\title{
Artificial Aggrecan prodrugs in the treatment of Arthrosis
}

\author{
Pardis Tabaee Damavandi \\ Queen Mary University of London
}

Author note: Copyright: $(\odot$ the author(s), publisher and licensee. This is an open-access article distributed under the terms of the Creative Commons Attribution Non-Commercial License, which permits unrestricted noncommercial use, distribution, and reproduction in any medium, provided the original work is properly cited. 


\begin{abstract}
Arthrosis (OA) is a debilitating disease which increasingly affects the geriatric population. Recent studies have shown that proteoglycan forming cartilage in human tissue is citrullinated in osteoarthritic patients and this could be the cause for the enhanced fragility in the knee joints, leading to fractures and falls. The flexibility of citrulline could be reduced compared to that of arginine as citrulline presents incorporations of rigid nitric elements as well as "condensed" proline atoms, making it resemble a metabolic byproduct of collagen and arginine. In truth, citrulline derives from the activity of multiple enzymes, both aggrecanase and arginase- 1 . The commentary describes causal involvement of citrullination in $\mathrm{OA}$ and also indicates potential therapeutic approaches to how displacing the pathological citrullinated arginine, could help patients with OA.
\end{abstract} Keywords: Arthrosis, Citrullination, Proline, Aggrecan, Drug design 


\section{Artificial Aggrecan prodrugs in the treatment of Arthrosis}

Arthrosis, also known as osteoarthritis (OA), is a peculiar and common type of arthritis that affects the geriatric population, and is caused by a degeneration of cartilage which sustains the organism, together with the remainder of the skeletomuscular system. Arthrosis per se is often used to describe the deterioration of the cartilage at the knee joint, where the articular cartilage that surrounds the patella is eroded through aging.

Currently, there is no cure for arthrosis, nor for rheumatoid arthritis (RA) or the other three types of arthritis, but some approaches involve invasive practices, such as microfracture procedures ${ }^{1}$, which however cause fibrosis instead of stimulating cartilage growth, autografting (to prevent rejection), and finally chondrocyte or stem cell implantations within the affected site.

The main challenge is that cartilage formation is impaired in the aging adult, and there are many hypotheses why this could be, however, the shared denominator is the compromise of the materials that compose the cartilage itself.

Cartilage is mainly made of proteic elastic molecules and thus has the ability to resist changes of tension and other forms of pressure thanks to its primary content of proteoglycans $\left(\right.$ aggrecan $\left.^{2}\right)$, hyaluronan and collagen. Chondrocytes and cartilage form networks of linking proteic structures that resemble "nets of quasi super-resistant fibers".

Aggrecan $^{3}$, whose heterogeneity has been discovered in patients suffering from both OA and RA, is "affiliated" structurally with collagen, also playing an important role in the development of arthrosis. Further, we explain why aggrecan is "familiar" to collagen, but substantially aggrecan is citrullinated ${ }^{4}$ in place of arginine in patients with RA. It is the citrullination of aggrecan that has been revealed inducing an immune response in RA, but its degradation-citrullination is not required to get an immune response, it can also be depicted whereby arthrosis is concerned (OA 
does not necessitate an immune response as it happens in RA instead). Intuitively, it can moreover be demonstrated that structural changes in the aggrecan structure, through its pathological citrullination are relevant in the pathogenesis of Arthrosis as functionality, as predicted by QSAR analysis often carried out by computational medicinal chemists, is strictly linked to the structure of aggrecan, by a mere definition of structure-activity relationship. Lastly, strategies will be discussed towards alternative therapy and directions of future research by the scientific community.

\section{Commonalities between the citrullinated aggrecan and collagen structures}

Collagen as we all know is made of three essential amino acids, hydroxyproline (a precursor of proline), proline and glycine. Several studies show that the impact of obesity on arthrosis other than producing strain on the kneecap due to the increase of the patient's mass, also is linked with an increment of glycine degradation, which plays an indirect role in arthrosis.

Citrullinated aggrecan too, is familiar in structure to collagen, and it looks as if hydroxyproline and proline play a role in the citrullination of aggrecan itself, since citrulline looks like a "compressed and metabolised" version of proline and hydroxyproline, since it is a highly carbonylated, highly argininated-nitrated form of hydroxyproline, proline and aggrecan. It appears as a synthetic "combination-biosynthesis", pathological in nature, of the collagen and aggrecan structures. This citrullinated structure inserts itself in the HLA-DRB1 pocket and rests within that very $\beta$ sheet tunnel. Studies have shown that in truth, citrullination is carried out by the Arginase1 enzyme, although the work is contrasted by RA research which highlights how citrullination causes the formation of epitopes that evoke auto-immunity ${ }^{5-9}$. It is possible that proline may play a role in the incorporation within the aggrecan molecule. This could be supported by the fact that native aggrecan, lacking in citrulline is not recognized by the immune response and remains unattacked. Nitric oxide (NO) reduction due to iatrogenic impairment could also lead to a 
disequilibrium in the arginine-citrulline ratio. Citrulline in its ramified and carbonated state, predictively looks less elastic/flexible than arginine, and this could explain fragility in the cartilage of the patients with citrullinated aggrecan. Further studies can be carried out to calculate the effective flexibility of citrullinated aggrecan against aggrecan. ${ }^{10}$

\section{Therapeutic strategies}

Heavy metals are known to have a particular type of trophism towards bone, as well as uric acid (as it happens in gout) and allopurinol, which is the notorious drug administered to displace uric acid. One approach could involve displacing citrullinated aggrecan with healthy aggrecan, without invasive procedures. This could be achieved by 1) simply designing a prodrug of the aggrecan structure, wherewith both the valines and tyrosine amino acids in the "un-citrullinated" aggrecan are $\mathrm{N}$-acetylated (esterified) and O-phenoxylated (and even O-acetylated), respectively, or 2) the arginines in the un-citrullinated aggrecan are acetylated, or 3) blending the two strategies, ultimately all leading to the administration of the "pro-drugged" aggrecans to the patient.

Another option could be the integration of a proline-rich diet of the geriatric population, which is normally present in broccoli, strawberries, asparagus etc.

\section{Conclusion}

Citrullinated aggrecan, an altered proteoglycan, has demonstrated having a relevant role in the pathogenesis of both Osteoarthritis and Rheumatoid Arthritis, whilst the non-citrullinated form is absent in the geriatric population due to both proteoglycan and collagen being at their physiological state, incising on their "normal" functions. Artificial prodrugs of uncitrullinated aggrecan could be designed to be administered on patients suffering from OA or RA as a way to displace the defective citrullinated-aggrecan forms. Proline rich foods can also be included in the diet of the geriatric population. 
No conflicts of interest to disclose. 


\section{References}

1. Shin, J. J., Mellano, C., Cvetanovich, G. L., Frank, R. M., \& Cole, B. J. (2014). Treatment of glenoid chondral defect using micronized allogeneic cartilage matrix implantation. Arthroscopy techniques, 3(4), e519-e522. https://doi.org/10.1016/j.eats.2014.05.014

2. Roughley, P. J., \& Mort, J. S. (2014). The role of aggrecan in normal and osteoarthritic cartilage. Journal of experimental orthopaedics, 1(1), 8. https://doi.org/10.1186/s40634014-0008-7

3. Stephen W. Scally, Jan Petersen, Soi Cheng Law, et al.; A molecular basis for the association of the HLA-DRB1 locus, citrullination, and rheumatoid arthritis. J Exp Med 18 November 2013; 210 (12): 2569-2582. doi: https://doi.org/10.1084/jem.20131241

4. KIANI, C., CHEN, L., WU, Y. et al. Structure and function of aggrecan. Cell Res 12, 1932 (2002). https://doi.org/10.1038/sj.cr.7290106.

5. Meesters DM, Hannemann PF, van Eijk HM, et al. Enhancement of fracture healing after citrulline supplementation in mice. Eur Cell Mater. 2020 Mar 20;39:183-192. doi: 10.22203/eCM.v039a12.

6. Von Delwig A., Locke J., Robinson J.H., Ng W.F.. 2010. Response of Th17 cells to a citrullinated arthritogenic aggrecan peptide in patients with rheumatoid arthritis. Arthritis Rheum. 62:143-149. https://doi.org/10.1002/art.25064

7. P Pandey, A. K., Naduthambi, D., Thomas, K. M., \& Zondlo, N. J. (2013). Proline editing: a general and practical approach to the synthesis of functionally and structurally diverse peptides. Analysis of steric versus stereoelectronic effects of 4-substituted prolines on conformation within peptides. Journal of the American Chemical Society, 135(11), 43334363. https://doi.org/10.1021/ja3109664 MID: 32195554. 
8. Videman T, Eronen I, Candolin T. [3H]proline incorporation and hydroxyproline concentration in articular cartilage during the development of osteoarthritis caused by immobilization. A study in vivo with rabbits. Biochem J. 1981 Nov 15;200(2):435-40. doi: 10.1042/bj2000435. PMID: 7340843; PMCID: PMC1163554.

9. Huang K, Wu LD. Aggrecanase and aggrecan degradation in osteoarthritis: a review. J Int Med Res. 2008 Nov-Dec;36(6):1149-60. doi: 10.1177/147323000803600601. PMID: 19094423.

10. Pardis Tabaee Damavandi, Martin T. Dove \& Richard W. Pickersgill (2017) A review of drug therapy for sporadic fatal insomnia, Prion, 11:5, 293-299, DOI: $10.1080 / 19336896.2017 .1368937$ 